\title{
Challenges in the Delivery of Therapies to Melanoma Brain Metastases
}

\author{
Gautham Gampa $^{1}$ - Shruthi Vaidhyanathan ${ }^{1} \cdot$ Brynna Wilken-Resman ${ }^{1}$. \\ Karen E. Parrish ${ }^{1}$ - Svetomir N. Markovic ${ }^{2}$ - Jann N. Sarkaria ${ }^{3}$ • William F. Elmquist ${ }^{1}$
}

Published online: 9 November 2016

(C) Springer International Publishing AG 2016

\begin{abstract}
Brain metastases are a major cause of morbidity and mortality in patients with advanced melanoma. Recent approval of several molecularly targeted agents and biologics has brought hope to patients with this previously untreatable disease. However, patients with symptomatic melanoma brain metastases have often been excluded from pivotal clinical trials. This may be in part attributed to the fact that several of the approved small-molecule molecularly targeted agents are substrates for active efflux at the blood-brain barrier, limiting their effective delivery to brain metastases. We believe that successful treatment of melanoma brain metastases will depend on the ability of these agents to traverse the blood-brain barrier and reach micrometastases that are often not clinically detectable. Moreover, overcoming the emergence of a unique pattern of resistance, possibly through adequate delivery of combination targeted therapies in brain metastases, will be important in achieving a durable response. These concepts, and the current challenges in the delivery of new treatments to melanoma brain metastases, are discussed in this review.
\end{abstract}

Keywords Melanoma brain metastases $\cdot$ Blood-brain barrier $\cdot$ Drug delivery $\cdot$ Molecularly targeted agents $\cdot$ Drug resistance $\cdot$ Active efflux

This article is part of the Topical Collection on Molecular Drug Disposition

William F. Elmquist

elmqu011@umn.edu

1 Brain Barriers Research Center, Department of Pharmaceutics, University of Minnesota, 308 Harvard Street SE, Minneapolis, MN 55455, USA

2 Division of Hematology, Mayo Clinic, Rochester, MN, USA

3 Radiation Oncology, Mayo Clinic, Rochester, MN, USA

\section{Introduction}

Metastatic melanoma is the most lethal form of skin cancer, causing the majority of skin cancer-related deaths. Current estimates for the year 2016 are approximately 76,380 new cases of metastatic melanoma with 10,130 deaths in the USA alone $[12,120]$. The fact that melanoma accounts for less than $2 \%$ of skin cancer diagnoses represents a dire situation. Early diagnosis leads to a good prognosis with 5-year survival rates of over $90 \%$ for patients with localized melanoma [22]. This survival rate falls steeply in patients with late-stage diagnosis and clinically evident metastases [22].

Melanoma patients with visceral metastases have lower survival rates compared to those with localized metastases, e.g., sentinel lymph node disease. The most frequent sites of visceral metastases are the lung, liver, bone, and brain [21, 125]. An interesting observation is that the 1-year survival was higher when the only site of visceral metastases was the lung compared to other visceral metastases [125]. This could be an indicator of the importance of the metastatic microenvironment and/or drug delivery.

A frequent site of melanoma metastases is the brain. Up to $75 \%$ of melanoma patients develop brain metastases within 3 years of diagnosis, and melanoma brain metastases (MBM) are associated with a median overall survival (OS) of less than 6 months. Most of these patients eventually die from progressive disease. About $55 \%$ of all deaths in melanoma patients are due to the presence of MBM $[22,26,121,143]$. Also, the presence of MBM in 55-75\% of melanoma patients at autopsy indicates a severely unmet medical need $[51,80]$.

Brain metastases are difficult to treat due to limited surgical options, hindered drug delivery, and radiation effects on cognition, all of which lead to limited treatment options and poor OS. Patients with multiple brain metastases and extensive peripheral disease can have particularly poor survival, which can 
be as short as $1-2$ months $[54,103]$. Patients with one to three brain metastases are often treated with surgical resection or stereotactic radiosurgery, while those with several brain metastases typically receive whole brain radiation [42]. Even though over the course of the last decade the treatment of systemic (peripheral) melanoma has seen a tremendous progress, new treatment paradigms are definitely needed to improve outcomes for patients with MBM.

A basic shortcoming in the clinical development of treatments for melanoma is that several of the recently approved small-molecule drugs, such as the BRAF and MEK inhibitors, have been approved based on clinical trials that have excluded patients with symptomatic MBM. A review of the inclusion/ exclusion criteria of clinical trial data on clinicaltrials.gov indicates systematic exclusion of melanoma patients with brain metastases from several clinical trials testing novel therapeutic agents [26, 48, 121, 143]. Such exclusion criteria have led to a lack of clarity about the efficacy of recently approved drugs in the treatment of MBM. The aim of this review is to discuss the challenges encountered in the delivery of current therapies to address the dynamic complexity of MBM.

\section{Biology of Formation of Brain Metastases}

The interaction of metastatic melanoma cells with the bloodbrain barrier (BBB), the extracellular matrix (ECM), and the natural tropism of melanoma to the brain are a few important factors influencing the formation of micrometastases and establishment of brain disease [40]. The invasion and growth of tumor cells in the brain parenchyma are facilitated by the interaction of metastatic cells with the BBB endothelial cells and surrounding ECM components [39, 40, 64, 139]. The high percentage of brain metastases from advanced melanoma as compared to other solid tumors is suggestive of a possible brain tropism of metastatic melanoma [143]. The "seed and soil" hypothesis proposed by Stephen Paget tries to explain the dissemination of tumor cells ("seed") from the primary site via the bloodstream to distant organs based on an inherent biochemical affinity of cancer cells for a certain distant site ("soil") which further leads to the development of metastases $[38,39,72,108]$.

Metastasis is a multistage process where the cancer cells from the primary site disseminate to distant organs. The metastatic cascade in the formation of MBM is comprised of a series of sequential processes where tumor cells escape from the primary tumor, enter the blood circulation, bind to the endothelial cells of the brain microvasculature, and extravasate/cross the BBB into the brain parenchyma [41, $65,100,139]$. This is followed by invasion and interaction within the brain microenvironment leading to survival and proliferation of tumor cells. Based on the characteristics of the tumor cells, they can either grow by forming new blood vessels (angiogenesis) or develop by growing along preexisting blood vessels (vascular co-option) [39, 41, 90]. Once melanoma micrometastases are established in the brain, this process could repeat at each new micrometastatic site making it a whole-brain disease and causing localized treatment strategies to be less effective.

The ulceration of the primary tumor is the strongest independent predictor of development of MBM and drastically reduces survival. Ulceration could probably be a marker of increased propensity for hematogenous dissemination, ECM invasion, and development of MBM [34, 39, 41, 143]. However, further investigation is necessary to clearly elucidate the underlying molecular mechanisms for brain-specific tropism.

Even though the specific underlying interactions that lead to the formation of MBM are not fully known, there have been a few recent findings that relate to possible driving mechanisms. For example, the expression of the gene encoding PLEKHA5 in melanoma tumors has been found to increase the propensity of early formation of MBM [35]. The $\beta 1$ integrins are known to play a central role in regulating the switch of cells to active and migratory mode helping with the metastatic cascade. This may be an important aspect in the process of formation of MBM [10]. Also, in another study, it was found that tight junction protein claudin 1 was found to suppress the malignant phenotype in melanoma [63]. A deeper understanding of the driving mechanisms that control the formation of MBM will be valuable in both treatment and prevention.

\section{Treatment Options in Patients with MBM}

Magnetic resonance imaging (MRI) and computed tomography (CT) are techniques commonly employed for detection of brain tumors. Passive contrast agent (e.g., gadolinium)-enhanced MRI is more sensitive than $\mathrm{CT}$ and positron emission tomography (PET)/CT in the detection of brain tumors. However, image enhancement with large, hydrophilic contrast agents requires the breakdown of $\mathrm{BBB}$, which in turn will lead to tumor detection in advanced metastatic disease, while missing detection of early micrometastases that are behind an intact BBB [121].

Initial diagnoses and treatment response assessment have been challenging with current diagnostic tools. An indirect assessment of brain tumor burden using contrast-enhanced MRI and CT is not a reliable method capable of differentiating between tumor progression, pseudoprogression, and pseudoresponse. Newer quantitative and physiologic imaging biomarkers are being studied and are promising [95, 98]. However, they have not yet made a positive impact to the field of neuroimaging to an extent that will be of general benefit to 
patients with CNS tumors. Identifying markers that predict response early in the disease progression may guide the process of drug selection and help clinicians make better treatment decisions. The possible use of blood-based biomarkers for CNS tumor assessment is also being actively investigated [61].

The therapeutic interventions employed for the treatment of MBM include surgery, radiation, and a combination of these with systemic therapies, both immunotherapy and chemotherapy $[75,108,110]$. Surgical resection is a preferred treatment option when feasible. In patients having solitary MBM in operable locations with a diameter of lesions over $3 \mathrm{~cm}$, surgical resection is appropriate as it appears to prolong survival $[70,75,110]$. For smaller lesions with size less than $2 \mathrm{~cm}$, or when the lesions are surgically inaccessible, stereotactic radiosurgery (SRS) is employed as an alternative. However, melanoma is a radiation-resistant tumor and also radiation is known to increase the risk of bleeding in brain lesions [11, 75, 110]. Whole-brain radiation therapy (WBRT) is considered for treatment when the patient has multiple MBM or for post-surgery/SRS control [46]. Systemic therapy would also be considered in the event of multiple systemic metastases at initial diagnosis [117].

The last decade has seen a dramatic improvement in the treatment of metastatic melanoma with the approval of molecularly targeted agents and immunotherapies. There is a marked interest in the recently approved molecularly targeted therapies, and the clinical usefulness of these agents for treating MBM is being actively investigated [108, 110]. However, a durable response is yet to be observed with regard to treatment with small-molecule chemotherapeutic agents. An important reason for lack of clarity in clinical outcomes can be attributed to the fact that several of the pivotal clinical trials excluded patients with MBM. This may be attributed to the poor survival rates and complications associated with MBM treatment.

\section{Systemic Therapies}

\section{Treatments from the Previous Era}

Before the advent of molecularly targeted agents and immunotherapies, the standard therapy for metastatic melanoma was the alkylating agent dacarbazine, which resulted in a poor median survival of 5-6 months and was accompanied by severe toxic side effects [114]. The second option, high-dose interleukin-2 (IL-2), has a poor response rate of less than $5 \%$ in patients with MBM and had significant toxicities including arrhythmias, hypotension, capillary leak syndrome, and severe neurological changes, thus making it a difficult treatment option $[8,9,53]$. Temozolomide therapy, another alkylating agent capable of crossing the $\mathrm{BBB}$, results in a poor survival rate of $7 \%$ and a progression-free survival of 1.2 months in MBM patients with no prior treatment [4].

In the past few years, tremendous progress in the understanding of genetic drivers of melanoma has led to the development and FDA approval of molecularly targeted smallmolecule agents (e.g., vemurafenib, dabrafenib, trametinib, and cobimetinib) for the treatment of metastatic melanoma. However, the success of these agents in treating melanoma metastases in the brain depends upon the delivery across the $\mathrm{BBB}$ and to the micrometastases that reside behind an intact BBB $[3,90]$.

\section{Deregulated Signaling Pathways and Molecularly Targeted Agents}

Oncogenic driver mutations in BRAF and NRAS of the mitogen-activated protein kinase (MAPK) signaling pathway, NF1 mutations, p53 mutations, and PTEN mutations have been identified to play key roles in the progression of melanoma $[13,60]$. Novel therapies that target specific molecular drivers of cancer progression such as the MAPK and phosphoinositide 3-kinase (PI3K)/mammalian target of rapamycin (mTOR) signaling pathways have brought new hope for improved treatment of metastatic melanoma in recent years. Figure 1 depicts the complex signaling cascade relevant to the small-molecule chemotherapeutics that can be used in the treatment of melanoma and their key targets.

The MAPK signaling pathway is deregulated in about $80 \%$ of melanomas as well as in many other human cancers [25]. Normally, signaling via the MAPK pathway requires the binding of a growth factor, cytokine, or hormone to a receptor tyrosine kinase which leads to the activation of RAS. RAS is upstream of RAF and upon activation recruits RAF to the cell membrane which further signals via downstream MEK and ERK kinases leading to cell growth and proliferation. Activating mutations lead to constitutive signaling via the MAPK pathway that triggers nuclear translocation of phosphorylated ERK and subsequent gene transcription, leading to uncontrolled cell growth and proliferation [78, 111].

An important therapeutic target in this RAS-RAFMEK-ERK signaling cascade is a serine-threonine kinase BRAF, an isoform of RAF [137]. A sequencing effort in 2001 identified important point mutations in the BRAF gene, and further genetic analysis led to the finding that point mutations in BRAF are the most commonly found somatic mutations in melanoma with a prevalence of approximately $50 \%$ [25, 57]. About $90 \%$ of all identified BRAF mutations result in a $\mathrm{V} 600 \mathrm{E} / \mathrm{D} / \mathrm{K}$ amino acid substitution which increases BRAF protein catalytic activity by approximately 50 200-fold as compared to wild type, resulting in constitutive activation of the MEK and ERK downstream proteins [25, 67, 137]. 


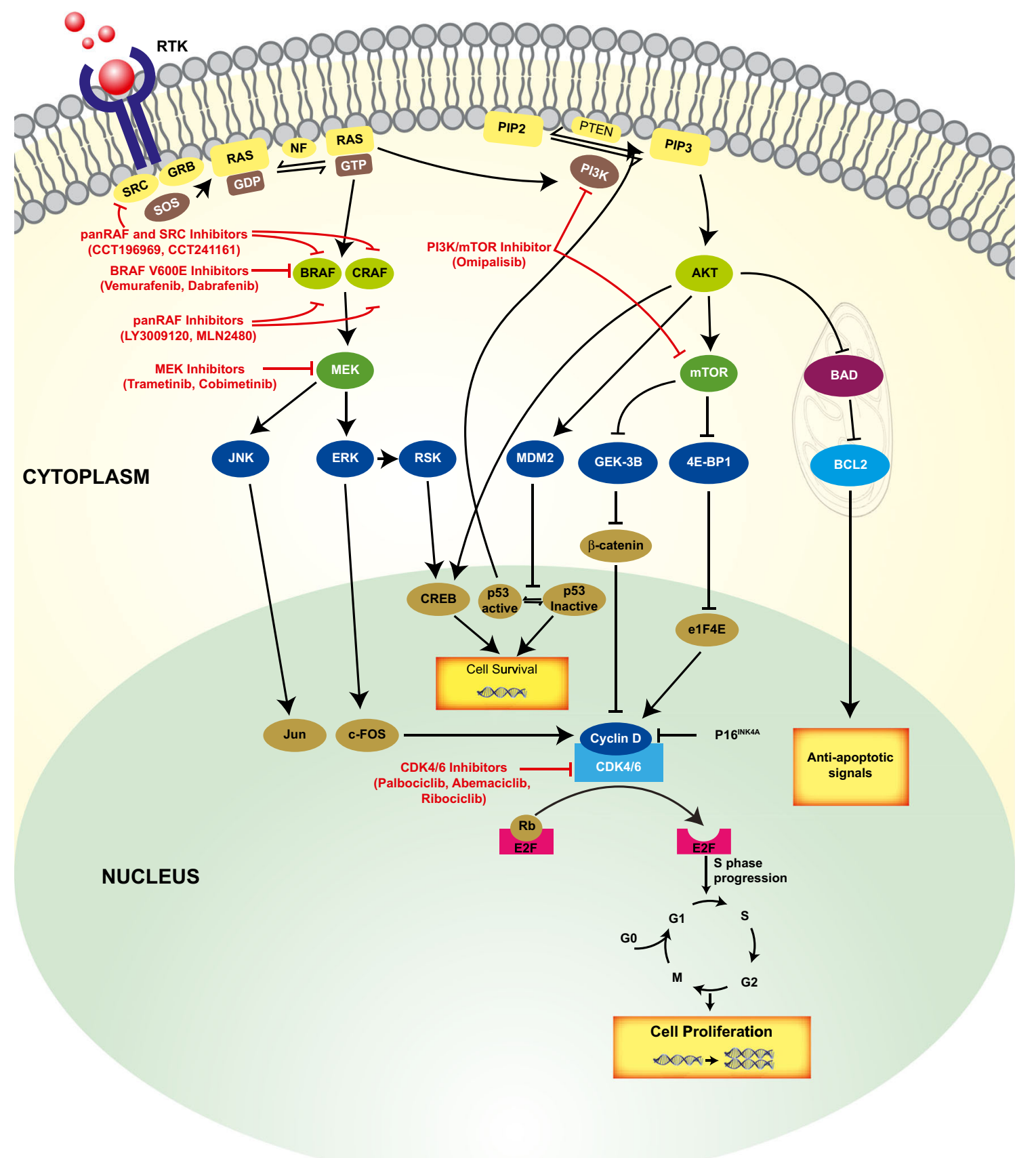

Fig. 1 Signaling pathways and small-molecule therapeutic targets relevant to current melanoma therapy. RAS/RAF/MEK/ERK (MAPK) and $\mathrm{PI} 3 \mathrm{~K} / \mathrm{AKT} / \mathrm{mTOR}$ (PI3K) pathways are the main signaling cascades linked to melanoma. The illustration represents the various

\section{BRAF-Targeted Therapy}

Vemurafenib (PLX4032) is a highly selective, potent, and well-characterized small-molecule BRAF V600E/D/K inhibitor that was developed using a structural-biology guided drug discovery approach [130]. The FDA approved it in August 2011 for patients with late-stage melanoma harboring the V600E mutation. Vemurafenib showed a remarkable mediators and regulators that are involved with melanoma pathogenesis. The key targets for various classes of small-molecule inhibitors are also shown

improvement in OS and progression-free survival (PFS) in a significant percentage of BRAF V600E-positive melanoma patients $[15,106]$.

Like vemurafenib, dabrafenib (GSK2118436) is a potent ATP-competitive inhibitor of the BRAF kinase, the second BRAF inhibitor to be approved by the FDA after it showed comparable clinical response to that of vemurafenib $[15,55]$. The safety and clinical response 
of dabrafenib against peripheral melanoma metastases were greater than $50 \%$ [55].

\section{Resistance Mechanisms}

Much of the current clinical data suggests that patients on BRAF inhibitors eventually stop responding to therapy due to the development of resistance and relapse of disease [99]. A resistance mechanism commonly associated with BRAF therapy has been the paradoxical activation of the MAPK pathway [96]. Mutations in upstream signaling proteins such as RAS or compensatory signaling from other growth factor receptors such as $\mathrm{PI} 3 \mathrm{~K} / \mathrm{mTOR}$ may be driving the reactivation of the MAPK signaling and strengthening the resistance to BRAF therapy [43]. Mechanisms suggested for development of resistance to BRAF therapy include one or more of the following: activating mutations in NRAS, upregulation of upstream RAS protein or platelet-derived growth factor receptor (PDGFR), increased PI3K/mTOR signaling, loss of PTEN, upregulation of cyclinD1, and downregulation of p27Kip1 [6]. Activating mutations in NRAS could potentially lead to signaling via CRAF, circumventing the inhibition of BRAF. Also, the loss of PTEN is commonly found in melanoma that results in the increased signaling via the PI3K pathway [134]. The occurrence of PTEN mutations with the concomitant maintenance of BRAF suggests that the PI3K pathway may be a potential driver of resistance.

A rational combination of drugs (small molecule and immunotherapies) that target multiple signaling pathways may eventually be the standard of care for the successful treatment of melanoma. However, in the context of treating MBM effectively, the challenge is the sufficient delivery of all agents in the combination across the $\mathrm{BBB}$ to the target sites in the brain that may reside behind an intact $\mathrm{BBB}$. Several new drug molecules are currently in development in combination with BRAF inhibitors such as RAS, MEK, and $\mathrm{PI} 3 \mathrm{~K} / \mathrm{mTOR}$ inhibitors [86].

\section{MEK Inhibitors}

In the context of delaying resistance, a recent success story is that of the orally bioavailable MEK (downstream on the MAP kinase pathway, i.e., MAP2K1, MAP2K2) inhibitor, trametinib, which was FDA approved as a single agent after it showed a 4.3-month PFS as compared to 1.5 months in the chemotherapy arm in phase 3 clinical trial in patients with BRAF V600E mutation [44].

The combination of BRAF inhibitor dabrafenib and MEK inhibitor trametinib has shown significant improvement in PFS in melanoma patients (9.4 months in the combination arm as compared to 5.8 months on monotherapy) [43] which led to the FDA approval of the combination. Later, in 2015, the FDA approved a second MEK inhibitor, cobimetinib, in combination with vemurafenib when the combination led to a significant improvement in PFS in vemurafenib-naive patients as compared to vemurafenib alone [73, 104].

However, acquired resistance to these combinations is a significant problem that needs to be addressed as a top priority in the search for a durable response [135]. In the case of the dabrafenib/trametinib combination, a case study looking at whole-exome sequencing (WES) and whole-transcriptome sequencing (RNA-seq) in five patients who had acquired resistance to the combination led to the identification of activating MEK2 mutations in three of the five patients $[135,136]$. This mutation was found to confer a profound resistance to the combination of dabrafenib/trametinib [136].

\section{PI3K/AKT/mTOR Pathway Inhibition}

It is observed that the PI3K/AKT/mTOR pathway is frequently altered by a functional loss of PTEN or the amplification of AKT [50]. Simultaneously, targeting of both the MAPK and the PI3K signaling is an approach that has been proposed and is actively being pursued in a subset of NRAS mutant melanomas [122].

The PI3K signaling is downstream of the RAS signaling molecule. PI3K phosphorylates a second messenger, phosphatidylinositol-4,5-biphosphate, thereby generating phosphatidylinositol-3,4,5-triphosphate, which activates AKT. Activated AKT has several cellular and enzymatic substrates that promote cellular proliferation, growth, and survival. In this context, omipalisib (GSK2126458), a potent and selective inhibitor of PI3K and the mTOR, was considered for combination therapy, but the clinical trial was terminated for toxicity issues (NCT01248858). Other signaling pathways driven by AKT and cyclin D-cyclin-dependent kinases 4/6 (CDK4/6) complexes are being investigated intensely to identify novel targets that can improve combination therapy and delay resistance [82].

\section{CDK4/6 Inhibitors}

The MAPK pathway and cell cycle checkpoint deregulation are frequently associated with NRAS mutant melanoma. Activation of the MAPK pathway can cause upregulation of cyclin D causing activation of CDK4/6 and cell cycle progression. This offers rationale to believe that simultaneous inhibition of MEK and CDK4/6 could have a synergistic effect in suppressing pathway activation and cell cycle progression [7]. A CDK4/6 inhibitor, abemaciclib (LY2835219), exhibited anti-tumor activity in a vemurafenib-resistant xenograft model [142]. In KRAS xenograft models, combination MEK and CDK4/6 inhibitor therapy, was found to be more effective than either agent alone [74]. 


\section{Pan-RAF Inhibitors}

Reactivation of the MAPK pathway via various mechanisms including paradoxical activation by BRAF inhibitors has made it challenging to treat patients with melanoma. The recently developed pan-RAF inhibitors have shown potential to inhibit all isoforms of RAF with minimal paradoxical activation by inhibiting both homo and hetero dimers of BRAF and CRAF [20, 49, 85, 92]. The pan-RAF inhibitor LY3009120 exhibits anti-tumor activities in KRAS, NRAS, or BRAF mutant tumors [92]. CCT196969 and CCT241161 are pan-RAF inhibitors that also inhibit SRC family kinases (SFK). They could be active as first-line treatment for both BRAF and NRAS mutant melanomas and also as second-line therapy for relapsed patients who were treated with BRAF and BRAF/MEK inhibitors [49]. Recent in vitro studies suggest that combination of pan-RAF and MEK inhibitors can cause complete inhibition of the MAPK pathway and could prove to be effective in NRAS mutant melanomas that are highly dependent on MAPK signaling [7, 85, 138].

\section{Targeting Immune Receptors}

Immune checkpoint blockade with antibodies has also been an important strategy that has been pursued to obtain durable responses in the treatment of metastatic melanoma. Antibodies directed against cytotoxic $\mathrm{T}$ lymphocyteassociated antigen 4 (CTLA-4), programmed death-1 (PD1), and programmed death ligand-1 (PD-L1) have shown promising response in a subset of melanoma patients [24, 140]. The FDA has approved four new immunotherapies, as monotherapy and in combination (ipilimumab, nivolumab, ipilimumab and nivolumab combination, and pembrolizumab), for advanced melanoma within the last 5 years. Immunotherapies for advanced melanoma fall into the categories of immune checkpoint inhibitors, oncolytic viruses, and cytokines. Current understanding of the efficacy and safety of these immunotherapies for patients with MBM is incomplete and is mainly derived from smaller clinical trials or retrospective analysis as these patients are often excluded from larger trials.

\section{Checkpoint Inhibition}

Treatment with immune checkpoint inhibitors leads to activation and proliferation of $\mathrm{T}$ lymphocytes that are capable of attacking malignant tumor cells. Activated $\mathrm{T}$ cells can gain entry into the CNS, providing the rationale for treating brain metastases with immune checkpoint inhibitors $[30,36]$. CTLA-4, a checkpoint protein expressed on $\mathrm{T}$ cells, competitively binds to ligands on antigen-presenting cells (APC) in order to suppress activation and proliferation of $\mathrm{T}$ cells (Fig. 2). Ipilimumab
(Yervoy, Bristol-Myers Squibb) is an anti-CTLA-4 monoclonal antibody and was the first immune checkpoint inhibitor to be approved by the FDA in 2011 . Ipilimumab showed an improvement in OS in two phase III clinical trials in melanoma patients [58, 107]. Tremelimumab, a second anti-CTLA-4 antibody, did not show a statistically significant improvement in OS [105].

The success with targeting CTLA-4 led to other approaches targeting immunological checkpoints. PD-1, expressed on activated $\mathrm{T}$ cells, and its ligand PD-L1 are negative regulators of $\mathrm{T}$ cell-based immune response. When engaged by its ligand such as PD-L1, PD-1 leads to suppression of immune response by causing inactivation of $\mathrm{T}$ cells and loss of effector function. Broadly, there are two categories of antibodies that target the PD-1/PD-L1 axis: antibodies that inhibit PD-1 and those that target and inhibit PD-L1 (Fig. 2). Nivolumab (Opdivo, BristolMyers Squibb) and pembrolizumab (Keytruda, Merck), approved by FDA in 2014, are PD-1-targeting antibodies that have shown durable responses in phase I clinical trials in patients with solid tumors $[127,128]$. The nature of the immune system to adapt to the changing tumor microenvironment may be advantageous in attacking the tumor cells. This provides a rationale for the durable responses seen with immunotherapies, as opposed to the responses observed with molecularly targeted agents due to acquired mutations [56, 79, 88]. A promising approach is to combine CTLA-4 and PD-1/PD-L1 antibodies for patients who benefit from immunotherapy as these checkpoints are believed to play separate roles in regulating anti-tumor responses. A combination of ipilimumab and nivolumab was one such approach that has shown durable responses in melanoma patients [140]. It remains to be seen whether immunotherapy can be effectively combined with molecularly targeted agents to improve patient outcomes while keeping side effects under control.

\section{Other Treatments}

Concerns of cerebral edema and neurotoxicity have until recently precluded the use of high-dose (HD) IL-2, a cytokine, in patients with brain metastases. Although case studies and small trials of HD IL-2 $(n<10)$ in this population have recently suggested that it may be safe for patients with MBM, its efficacy in these patients has not been evaluated in a larger study.[18, 97]. Other cytokines, including interferon alpha, have also been used in melanoma, with limited success and marked toxicity [66]. Some clinical studies have also evaluated granulocyte-macrophage colony-stimulating factor (GM-CSF) as a single agent or as adjuvant 


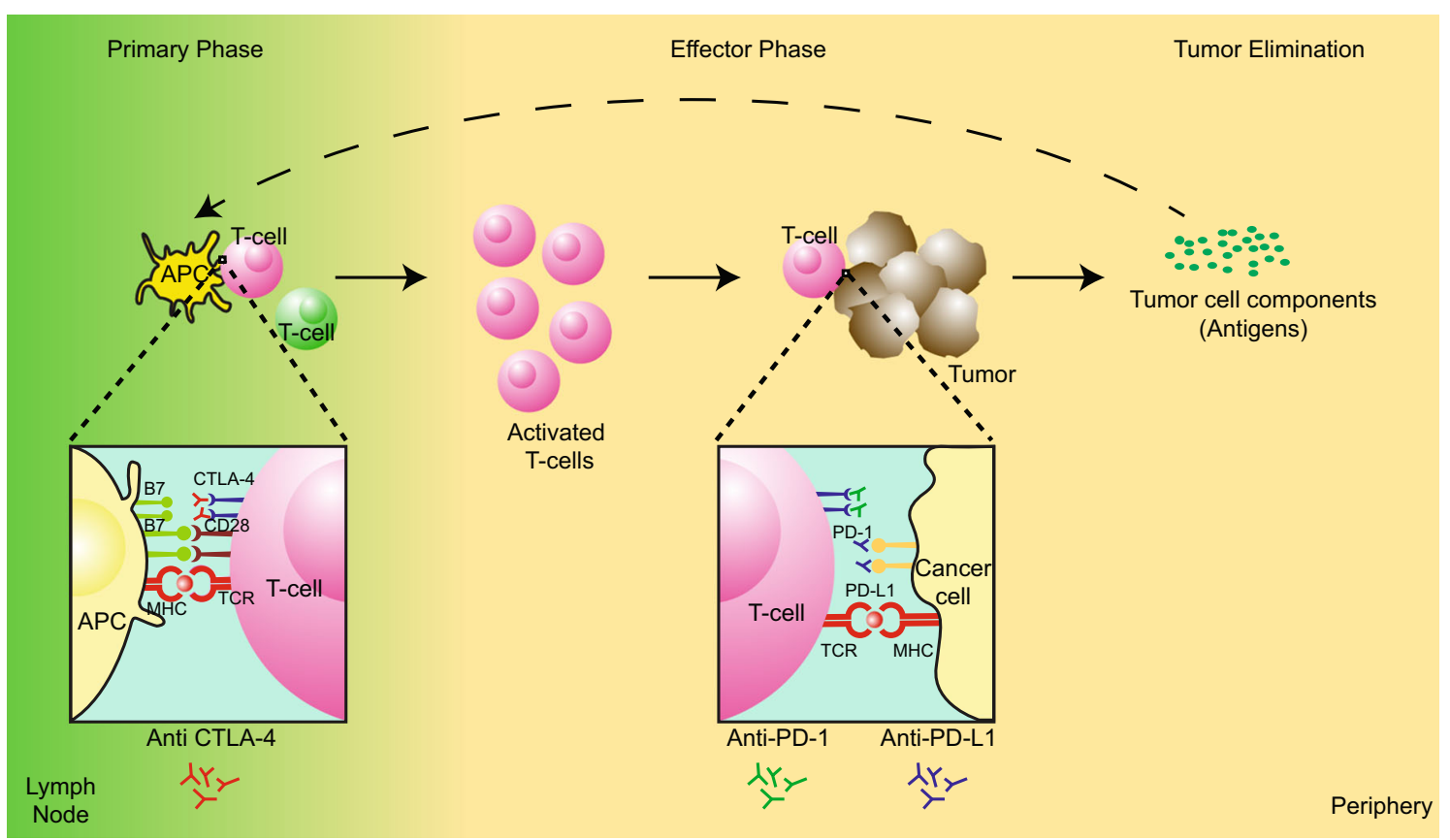

Fig. 2 Anti-tumor immune response through CTLA-4 and PD-1 pathway blockade and $\mathrm{T}$ cell signaling in the treatment of melanoma. Blocking CTLA-4 signaling and PD-1 signaling strengthens the

therapy with inconsistent results and unclear clinical benefit [68].

\section{The BBB Poses a Significant Challenge to Therapies}

\section{BBB as a Significant Barrier to Successful Treatment of MBM}

The brain microvasculature is lined by a continuous endothelium consisting of cells closely held together by tight junctions (zonula occludens) and surrounded by ECM components, pericytes, and astrocyte foot processes, which together form the neurovascular unit, i.e., the BBB [1]. This intricate structure shields the brain from many xenobiotics, including a large variety of drug molecules. In addition, the BBB is also fortified by the presence of numerous and diverse transport systems belonging to the ATP binding cassette $(\mathrm{ABC})$ and the solute carrier (SLC) families of proteins expressed on the basolateral and apical surface of the endothelial cells, and plays a critical role by causing influx and efflux of therapeutic agents used to treat brain diseases $[1,126]$. The reader is directed to the article by Abbott et al. for a detailed understanding of the BBB.

Two of the $\mathrm{ABC}$ efflux transporters appear to be particularly important in limiting drug delivery to the brain. Pglycoprotein (P-gp, ABCB1, MDR1), a 170-kDa phosphorylated glycoprotein, and breast cancer resistance protein activation of anti-tumor $\mathrm{T}$ cell response leading to tumor elimination. The activated $\mathrm{T}$ cells are able to gain entry into the brain (not shown in the figure) and act at the site of brain metastases

(BCRP), a 72-kDa half-transporter protein, are two highly expressed efflux transporters at the human BBB and are known to influence the CNS distribution of several drugs [2, 131]. The influence of these two efflux transport proteins is known to play a crucial role in the treatment of CNS diseases such as HIV and glioma [3, 87, 116]. In preclinical studies, there is strong evidence for the interaction of vemurafenib, dabrafenib, trametinib, palbociclib, cobimetinib, and omipalisib with P-gp and BCRP [17, 27, 33, 83, 84, 89, $132,133]$. The brain concentrations achieved by most of these drugs are less than $10 \%$ of their plasma concentrations (Table 1), and thus, it is less likely that therapeutic brain concentrations will be achieved before off-site toxicity in the periphery limits dose escalation. Active efflux at the BBB, mainly by P-gp and BCRP, is a key reason for lack of brain delivery of many therapeutic compounds $[2,32]$.

Also, melanoma cells that have been previously treated with chemotherapeutic agents have been known to express the ATP-dependent efflux transporter ABCB5, while a side population of stem cells from melanoma tumors are known to express $\mathrm{ABCB} 1$ on the plasma membrane and $\mathrm{ABCB} 5$ intracellularly $[16,76]$. The lack of efficacy of chemotherapy in treating brain tumors may partly be attributed to the presence of such secondary barriers in the tumor cells.

The BBB in brain tumors has long been thought to be compromised, and delivery of chemotherapeutics to the tumor cells was believed to be uninhibited [47, 93, 124]. Such a misconception is due to the fact that contrast-enhanced MRI is frequently used as a diagnostic tool for visualizing brain 
Table 1 Small-molecule chemotherapeutic agents examined for treatment of melanoma and their substrate status reported as brain to plasma ratio $(B /$ $P)$ in mice

\begin{tabular}{|c|c|c|c|c|c|c|c|c|c|}
\hline \multirow[t]{2}{*}{ Chemotherapeutic } & \multirow{2}{*}{$\begin{array}{l}\text { Molecular } \\
\text { target }\end{array}$} & \multirow{2}{*}{$\begin{array}{l}\text { Clinical } \\
\text { status }\end{array}$} & \multirow{2}{*}{$\begin{array}{l}\text { Substrate } \\
\text { status } \\
\text { P-gp }\end{array}$} & \multicolumn{5}{|c|}{$B / P$ in mouse models } & \multirow[t]{2}{*}{ Reference } \\
\hline & & & & BCRP & $\begin{array}{l}\text { Wild-type } \\
\text { mouse } B / P\end{array}$ & $\begin{array}{l}\text { P-gp KO } \\
\text { mouse } B / P\end{array}$ & $\begin{array}{l}\text { BCRP KO } \\
\text { mouse } B / P\end{array}$ & $\begin{array}{l}\text { P-gp and BCRP } \\
\text { KO mouse } B / P\end{array}$ & \\
\hline Vemurafenib $^{\mathrm{a}}$ & $\begin{array}{l}\text { Mutant } \\
\text { BRAF }\end{array}$ & Approved & Yes & Yes & $0.012 \pm 0.004$ & $0.035 \pm 0.009$ & $0.009 \pm 0.006$ & $1.00 \pm 0.19$ & {$[33,84]$} \\
\hline Dabrafenib $^{\mathrm{b}}$ & $\begin{array}{l}\text { Mutant } \\
\text { BRAF }\end{array}$ & Approved & Yes & Yes & 0.044 & nd & nd & 0.25 & [83] \\
\hline Trametinib $^{\mathrm{a}}$ & MEK & Approved & Yes & No & $0.28 \pm 0.009$ & $1.53 \pm 0.57$ & $0.14 \pm 0.13$ & $2.45 \pm 1.3$ & [132] \\
\hline Cobimetinib $^{\mathrm{c}, \mathrm{d}}$ & MEK & Approved & Yes & No & $0.08,0.32$ & $1.1,10.8$ & $0.67,0.21$ & $1.1,6.2$ & [17] \\
\hline $\begin{array}{l}\text { Omipalisib } \\
\qquad(\text { GSK2126458) }\end{array}$ & $\mathrm{PI} 3 \mathrm{k} / \mathrm{mTOR}$ & Phase I & Yes & Yes & $0.06 \pm 0.02$ & $0.05 \pm 0.03$ & nd & $0.46 \pm 0.23$ & [133] \\
\hline Palbociclib $^{\mathrm{a}}$ & CDK4/6 & Approved & Yes & Yes & $0.2 \pm 0.07$ & $2.5 \pm 0.1$ & $0.4 \pm 0.1$ & $28 \pm 6$ & {$[27,89]$} \\
\hline
\end{tabular}

nd not determined

${ }^{\text {a }}$ Steady-state brain to plasma concentration ratios

${ }^{\mathrm{b}}$ Brain to plasma ratio of $\mathrm{AUC}_{0-4}$ post oral dose

${ }^{\mathrm{c}}$ Brain to plasma ratio of concentrations $1 \mathrm{~h}$ post oral dose

${ }^{\mathrm{d}}$ Brain to plasma ratio of concentrations $6 \mathrm{~h}$ post oral dose

tumors. In a contrast-enhanced MRI, brain regions with a leaky BBB, such as the tumor core, allow the contrast agent such as gadolinium [GAD]-DTPA (a 938-MW contrast agent) to diffuse into the brain parenchyma, indicating the tumor core location [90]. However, it is imperative to recognize that this technique can indicate the locations of leaky larger brain tumors and may be incapable of indicating smaller tumors that reside behind an intact BBB. Since the smaller brain metastases are not detectable by the current diagnostic techniques, they are referred to as "subclinical." It is plausible that the brain metastases become leaky only after they reach a certain size while the micrometastases are still protected by an intact $\mathrm{BBB}$. As a consequence, the clinical contrast-enhancing larger metastases with leaky BBB may have relatively uninhibited drug delivery while the subclinical protected micrometastases with an intact BBB still do not have adequate drug delivery (Fig. 3). It has been reported that the BBB in brain metastases larger than $0.25 \mathrm{~mm}$ in diameter are leaky [39]. Therefore, these ideas and concepts indicate that it is crucial to treat both the larger metastases and the protected micrometastases to attain durable responses in patients with MBM.

Though the last decade has witnessed the approval of essential molecularly targeted agents and immunotherapies that improved the treatment of metastatic melanoma, targeting MBM has been particularly challenging due to the location of the tumors in a protected CNS sanctuary. The failure of clinical trials that were designed to test novel drugs for brain tumors may be, in part, due to the presence of tumor cells that reside behind an intact BBB that escape treatment. A majority of therapeutic agents are limited in their ability to distribute to such locations, and this may be a key reason for therapeutic failure leading to poor prognosis in patients with MBM. As the novel systemic therapies continue to effectively control the extracranial tumors, the number of patients affected by brain metastases will continue to rise [46]. Patients treated with systemic treatments for extracranial metastatic disease may have an increase in the incidence of brain metastases possibly due to the limited brain distribution of these agents such as vemurafenib [84, 94, 108]. Also, as a consequence of such subtherapeutic drug exposure, there may be an eventual development of unique patterns of resistance in the brain. This highlights the importance of developing brain-penetrant drugs that are specifically designed to permeate an intact BBB and reach not only the larger, leaky brain tumors but also the protected micrometastases residing behind an intact BBB.

Despite the progress in the treatment of peripheral metastatic melanoma, the standard of care for patients with brain tumor metastasis remains surgery and radiation [52]. Ineffective delivery of chemotherapeutics to brain tumors along with the invasive nature of metastatic brain lesions and their ability to undergo frequent mutations are some hindrances that reduce effectiveness of systemic therapy and eventually lead to the development of resistance, a common trait of peripheral metastases especially in melanoma [129]. Furthermore, the use of these small-molecule chemotherapeutics in the clinic has shown conflicting results in effectiveness in brain metastases [108]. Newer novel drugs directed towards better molecular targets may extend patient survival by controlling peripheral disease, but the limited brain penetration will result in a pharmacological sanctuary within the brain for the tumor cells, which could cause a rise in the incidence of brain metastases (Fig. 4). This could possibly worsen the 


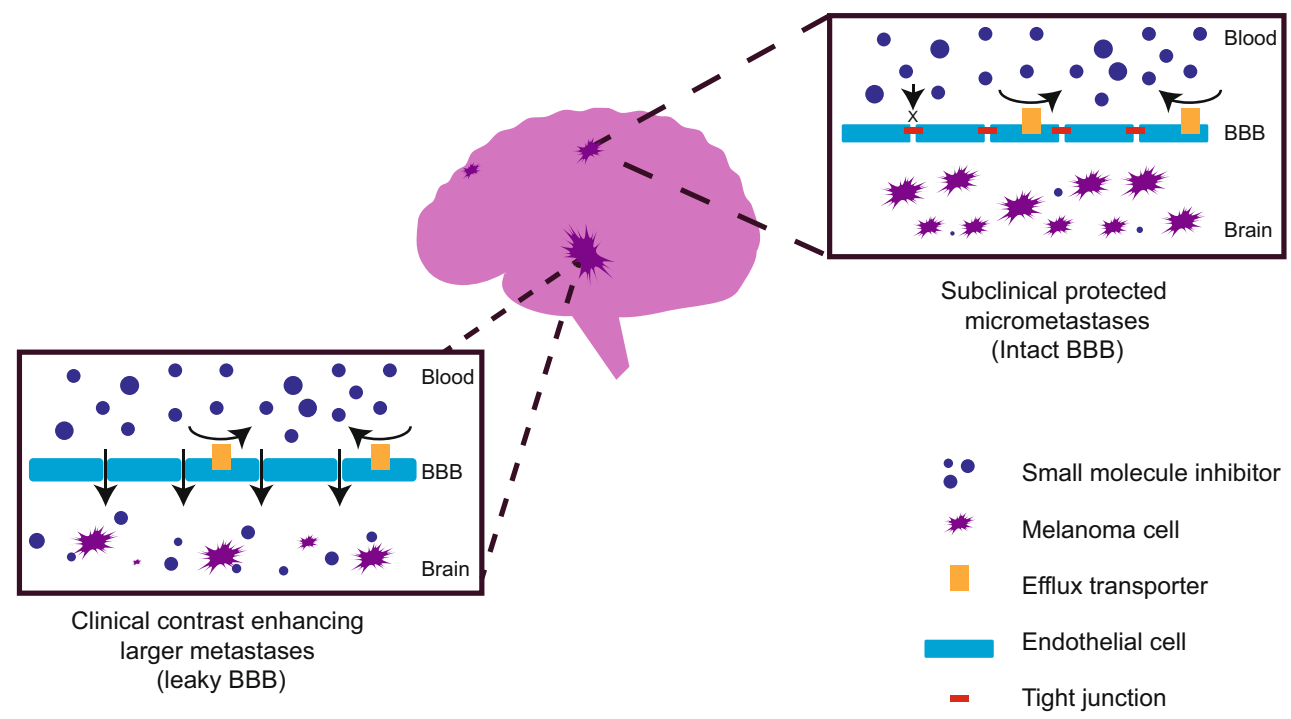

Fig. 3 Challenges with respect to drug delivery in treating melanoma brain metastases. The contrast-enhancing larger tumors in the brain have a relatively leaky BBB that allows distribution of drug molecules. However, the presence of subclinical "protected" micrometastases behind an intact $\mathrm{BBB}$, combined with the active efflux of drug molecules by efflux transporters at the BBB, may severely limit the delivery of anticancer agents to such "subclinical" tumors and will result in a lack of therapeutic efficacy disease, and thus, it is critical to focus on developing new therapeutic modalities that not only penetrate the BBB but also reach the intended target within the brain. If that goal is achieved, we may see a significant improvement in clinical outcomes. We also need to understand the mechanism by which the tumor cells migrate across the $\mathrm{BBB}$ to recognize how the circulating tumor cells may "seed" the brain in areas of an intact BBB (Fig. 5). Moreover, the tumor microenvironment could be constantly communicating with the tumor cells, playing an important role in its development. Metastases in the brain may behave very differently with regard to driver target evolution, expression of transporters, and therefore, response to treatment in the brain microenvironment versus peripheral sites (Fig. 6). Gaining such insights will provide a holistic understanding of the disease pathogenesis, which in turn will help us answer critical questions necessary to develop more effective therapies.

A useful MBM model for preclinical studies should be reproducible and recapitulate the human disease in that it should have tumors that metastasize from primary, peripheral

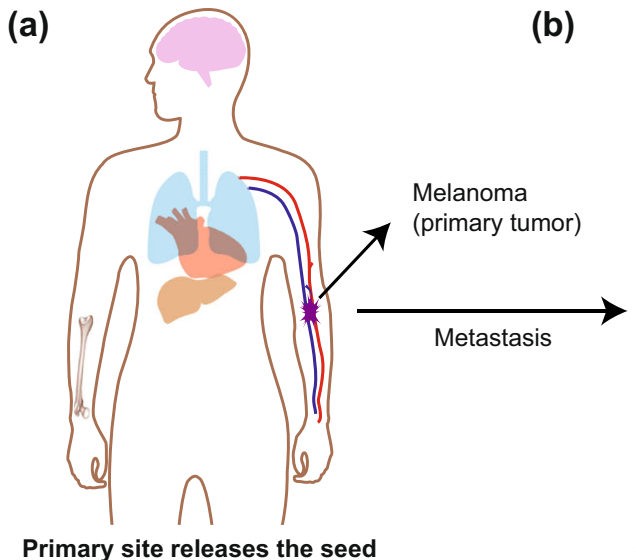

Primary site releases the seed

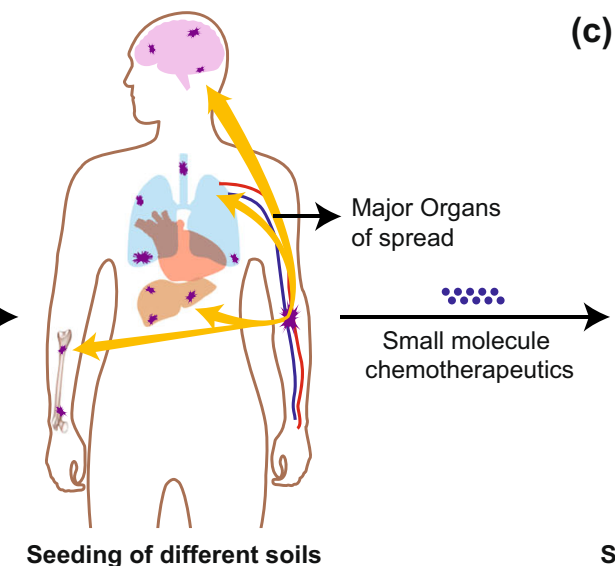

me soils are more amenable to drug delivery
Fig. 4 Metastatic spread of melanoma and a comprehensive outlook of small-molecule drug delivery to tumor sites. Panel a depicts a subject bearing a primary melanoma tumor. Panel $\mathbf{b}$ illustrates the spread of melanoma from the primary tumor site to the major organs of melanoma metastases. Panel $\mathbf{c}$ emphasizes the idea that the current chemotherapeutics, though potent and initially effective, are limited by their ability to distribute to the brain. Many small-molecule chemotherapeutics achieve therapeutic levels in the peripheral tumor locations, both primary tumors and metastases, outside the brain and can therefore initially elicit a positive response, before resistance is seen. However, limited drug distribution to regions of tumor in the brain can result in the establishment of a pharmacological sanctuary site, causing ineffective therapy 


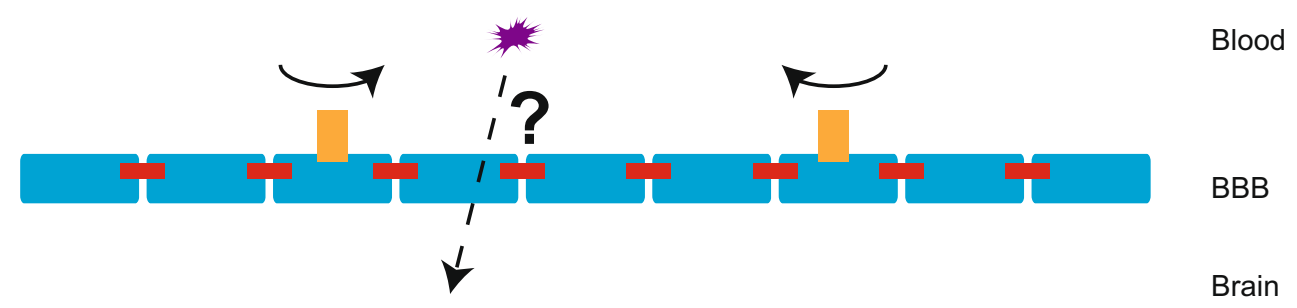

Blood

Brain

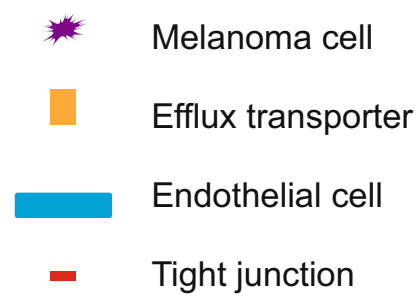

Fig. 5 This figure highlights that we do not fully understand the mechanism of extravasation of tumor cells to the brain from the peripheral primary tumors. A deeper understanding of the steps involved in the initial seeding of the metastatic tumor cells would provide insights into devising appropriate strategies to prevent the establishment of brain disease. The figure describes the conundrum of

sites with high proclivity to the brain, as seen clinically in patients with MBM [69]. Using unsuitable models to study metastatic brain tumors will result in misguided drug development efforts and poor translation to clinic. As an example, the protein expression in murine BBB has notable differences compared to that in human BBB. Relative P-gp and MRP4 expression are higher in mouse capillary endothelial cells than in human, whereas BCRP expression is higher at the human BBB [131]. Even so, our knowledge is still very limited about the species differences in terms of intrinsic activity of these transporters at the $\mathrm{BBB}$. The relative transport of drugs at the BBB will depend on both expression and activity of the efflux proteins. Other aspects of a useful model for testing therapeutic strategies for translation to the clinic include the choice of tumor cells. Importantly, patient-derived xenografts (PDX)

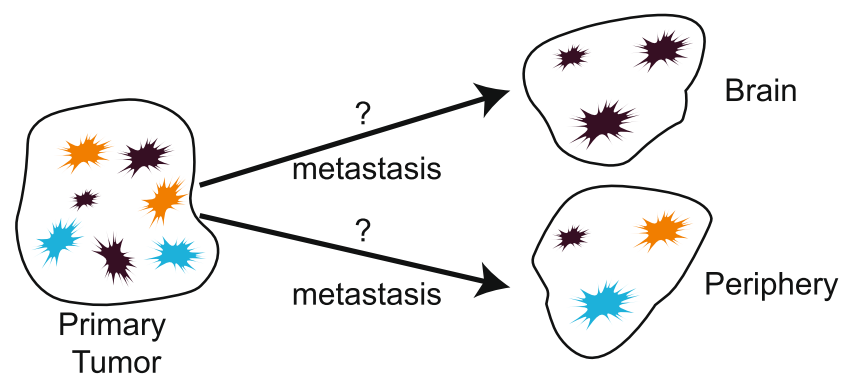

Fig. 6 The figure emphasizes that the way the tumor cells express targets or develop resistance could be different based on their microenvironment. It is possible that in the brain microenvironment, selection pressure is different than that in the periphery. This may cause distinct gene expression profiles by tumor cells in the brain versus the periphery, which could result in development of unique patterns of resistance in the brain how the circulating tumor cells enter the brain at sites that have an intact $\mathrm{BBB}$ and is side by side with the lack of drug penetration across that same intact BBB, one that includes both tight junctions and efflux transport. Such a problem would be seen in regions of larger metastases that have an intact BBB and undoubtedly in the micrometastatic sites early in the course of brain disease

maintain the same histopathology and genetic and molecular characteristics observed in the patient from whom they were derived [14]. Tumor cells derived from PDX models can be injected or implanted extracranially in immunodeficient mice, and allowed to develop MBM in order to study the efficacy of small molecule therapies. However, since they must be established in immunocompromised mice, they are not suitable for studying immunotherapies or the immune component of the disease. Genetically engineered mice models (GEMM) that harbor mutations common to MBM are another useful tool [109]. These improved models that are more similar in growth patterns of human disease should provide a better platform to study the efficacy of new treatments, including the influence of drug delivery.

Unfortunately, in spite of the recent advances in drug discovery for melanoma, the majority of therapies with potential for active efflux at the BBB may not achieve therapeutic levels in brain tissue, leading to ineffective therapy. Molecular mutations that cause unregulated tumor proliferation and growth are often preserved across many tumors. This leads to the design of target-based clinical trials that are conducted without sufficient understanding of delivery of the drugs to the target, especially the brain delivery of molecularly targeted agents. The hypothesis tested time and again is that the drugs showing therapeutic benefit in peripheral cancers will work in brain cancers. However, the clinical results have been routinely disappointing and do not support such a hypothesis. This shows that in vitro efficacy against tumor lines, and even in vivo efficacy against peripheral tumors in inappropriate animal models, is not sufficient to guide the development of experimental therapies for use in clinical trials for treating MBM. 


\section{Preclinical and Clinical Examples}

\section{Small-Molecule Chemotherapeutics}

An important factor that can contribute to the near $100 \%$ mortality in patients with MBM is likely to be the limited brain permeability of small-molecule therapeutic agents across an intact BBB, hindering delivery to subclinical metastases in the brain. In this context, it is important to review the role of BRAF inhibitors for the treatment of MBM in addition to surgery and radiation therapy. In the case of vemurafenib, prior evidence through case studies in the literature suggests that there has been a potential lack of efficacy in the treatment of MBM [108]. Conversely, dabrafenib showed a promising reduction in tumor size in $\sim 90 \%$ of the patients with MBM [37]. Also, vemurafenib was reported to be effective in MBM patients with $37 \%$ of patients achieving $>30 \%$ intracranial tumor regression and $16 \%$ achieving a confirmed partial response [median OS of 5.3 months] [31]. It is possible that such positive results could be seen due to the presence of larger leaky tumors in the patients evaluated. However, it is still not clear whether these drugs will be effectively delivered to micrometastatic sites, particularly those that are situated beyond an intact BBB. With this consideration, it is important to study the brain distribution of both these molecules in greater detail.

The combinations of BRAF and MEK inhibitors (dabrafenib/trametinib (GSK) and vemurafenib/cobimetinib (Roche)) are currently being tested in clinical trials for patients with melanoma that has metastasized to the brain. The primary purpose of the dabrafenib/trametinib study (NCT01978236) is to determine the distribution of these molecularly targeted agents in parenchymal MBM. The results of this study will indicate the distribution of dabrafenib and trametinib to resectable regions of MBM. However, the drug distribution to micrometastases that are undetected in the clinical setting will remain enigmatic. Our lab has demonstrated that dabrafenib has a greater brain to plasma distribution ratio than vemurafenib in preclinical studies conducted in mice [83]. This could be a possible explanation for the improved efficacy observed with dabrafenib. It was also observed that trametinib has a higher distribution ratio compared to vemurafenib and dabrafenib in preclinical models [83, 84, 132].

Preliminary evidence in patients with MBM suggests that combining dabrafenib and trametinib with SRS may lead to improved survival compared to dabrafenib alone [91]. A rational hypothesis based on local concentration-effect relationships suggests that the success of single-agent or combination therapy to delay/overcome resistance is related to the ability of individual agents to cross the $\mathrm{BBB}$ and reach micrometastatic sites. Currently, several clinical trials are investigating the efficacy and survival benefit of molecularly targeted agents in treating patients with MBM. It will be of the utmost importance, however, to recognize that the local drug concentrations sampled via surgical resection in these studies may not be those concentrations that reflect eventual therapeutic outcome.

A novel CDK4/6 inhibitor, abemaciclib (LY2835219), is currently in a clinical trial in patients with MBM (NCT02308020). It has been reported to have an enhanced brain distribution due to saturation of efflux transport at the BBB in preclinical models of glioma [141, 142]. While the ability of abemaciclib to enhance its own brain distribution remains to be further verified and shown in the clinical setting, this attribute could potentially allow an enhanced efficacy in MBM. Other critical studies include a phase $\mathrm{Ib} / \mathrm{II}$ clinical study of the combination of a MEK inhibitor binimetinib and a CDK4/6 inhibitor LEE011 (NCT01781572) [74]. Early clinical results are supportive of promising anti-tumor activity in patients with NRAS mutant melanoma [123]. This study has the severe drawback in determining efficacy in MBM, in that the study excludes patients with detectable brain metastases.

\section{Immunotherapies}

In a recent clinical trial, Margolin and colleagues evaluated the use of ipilimumab in 51 patients with active but asymptomatic MBM (cohort A) and 21 patients with symptomatic MBM (cohort B). Cohort A showed a $24 \%$ response rate in brain metastases and a $27 \%$ response rate in peripheral tumors. Cohort B showed lower response rates for both brain and peripheral metastases, possibly due to the immunosuppressive effects of corticosteroids [77]. Additionally, another phase II trial investigating the combination of ipilimumab and fotemustine enrolled melanoma patients with $(n=20)$ and without ( $n=66$ ) detectable brain metastases and found similar proportions of disease control between these two groups (50.0 and $46.5 \%$, respectively) [28]. Follow-up showed that the patients with brain metastases had long-term responses (2and 3-year PFS of 35 and $25 \%$, respectively) [29]. In another clinical trial, 146 patients with asymptomatic MBM were treated with single-agent ipilimumab. The median PFS and OS were 2.8 and 4.3 months, respectively. The 1 -year survival rate was about $10 \%$ [101]. Retrospective analysis for a number of clinical trials has also yielded information on the efficacy of ipilimumab in melanoma patients with brain metastases, as summarized by Ajithkumar and colleagues [5].

Pembrolizumab is currently being evaluated in patients with asymptomatic brain metastases not requiring steroids as a single agent for patients that have not yet received any prior treatments (NCT02085070). In addition to pembroliumab (anti-PD-L1), ipilimumab (anti-CTLA4) and nivolumab (antiPD-1) are currently undergoing clinical evaluation for the treatment of patients with MBM. It will be critical to understand the role of the immune system in regulating intracranial 
tumor growth and whether or not these agents need to enter the brain to elicit an effect, in order to predict the efficacy of these agents in the treatment of MBM. Previous studies have reported that ipilimumab and cranial radiation is well tolerated in MBM patients, and early studies have indicated that the timing of radiation and immunotherapy administration may be important to optimize outcomes [113]. A retrospective analysis of patients with metastatic melanoma receiving ipilimumab did not show a reduction in the development of brain metastasis in the ipilimumab-treated group when compared with vemurafenib-treated patients [45].

A case study that examined the use of pembrolizumab in treating a patient with MBM showed enhanced tumor burden after 11 days of pembrolizumab treatment. The authors report that the enhanced region was filled with microglial cells, inflammatory cells, and isolated clusters of tumor cells, all indicative of pseudoprogression [19]. In another case study, it was reported that an enhanced tumor burden was observed in a patient with MBM post-treatment with ipilimumab. A pathological review of resected brain metastasis revealed focal areas of necrosis with marked infiltration of the tumor by lymphocytes and melanophages [59]. The experience with such cases of pseudoprogression has been higher in patients with primary brain tumor, i.e., glioblastoma (GBM). In a study published by Sanghera et al., it was observed that GBM patients with pseudoprogression had a significantly longer median survival (124.9 weeks) compared to GBM patients showing disease progression (36 weeks) [112]. Additional studies report similar survival benefits in GBM patients with pseudoprogression [71, 102]. Mechanisms leading to a favorable prognosis in GBM patients with pseudoprogression could also be applicable in the context of MBM patients with pseudoprogression. Currently, there is no imaging technique that can reliably distinguish pseudoprogression from true progression [62]. As a result, there is a dilemma with respect to changes observed in brain tumor imaging early on in the disease that may be an indication of improved outcomes to treatment, or may turn out to be true progression.

\section{Dual Immunomodulation and Sensitization to Immunotherapy}

Given the success of immunostimulatory antibodies targeting CTLA-4 and PD-1, there is much interest in combining checkpoint inhibitors of multiple pathways to enhance the immune response and increase the proportion of patients who respond to treatment. The combination of ipilimumab and nivolumab was approved by the FDA in October of 2015. There is currently no clinical trial data specifically addressing efficacy of combination checkpoint inhibition in patients with MBM, but there are a number of ongoing trials, including a phase 3 clinical trial investigating efficacy of ipilimumab alone, ipilimumab with fotemustine, and ipilimumab with nivolumab in patients with brain metastases (NCT02460068 (NIBIT-M2)). Future combination therapies may include other co-inhibitory molecules such as PD-L1, LAG-3, TIM-3, VISTA, BTLA, and co-stimulatory molecules including CD40, OX40, CD137, and GITR, as well as cytokines and oncolytic viruses [81, 115, 118, 119].

There are also trials testing an immune checkpoint inhibitor with radiation or conventional anti-cancer drugs in order to provoke an immunogenic tumor microenvironment that can be more easily detected by immune surveillance, hence "sensitizing" the tumor to immunotherapy [118]. The case of President Jimmy Carter's remission from melanoma that had spread to both the liver and the brain, when treated with a combination of radiation therapy and pembrolizumab, is an example of combination therapy proving to be effective and brings new hope to treat this debilitating condition [23].

\section{Conclusions}

Despite the dramatic improvement in the treatment of metastatic melanoma with the approval of several novel molecularly targeted therapies and immunotherapies, the utility of these agents in treating MBM has been controversial and limited in duration. As these novel therapies initially control the peripheral disease, there may be a rise in the incidence of MBM. A major reason for difficulty in treating MBM could be attributed to the limited distribution of the molecularly targeted agents across the $\mathrm{BBB}$, due to the presence of tight junctions and efflux proteins. P-gp and BCRP, expressed on the luminal side of the BBB, are capable of actively effluxing various therapeutic agents used for treating melanoma. To achieve therapeutic success, the targeted agents should be delivered not only to the contrast-enhancing leaky larger tumors but also to the subclinical micrometastases protected by an intact BBB. Another important reason for lack of therapeutic success is development of resistance to therapies. Thus, it is imperative to develop brain-penetrant multitargeted agents or combination therapies that can be delivered effectively to the target sites, so that both delivery and resistance issues can be tackled. Incorporating these concepts in future clinical trials testing novel therapies will significantly enhance the progress towards a successful and durable treatment of MBM.

Acknowledgments This work was partially supported by National Institutes of Health grants from National Cancer Institute CA138437 (WFE) and National Institute of Neurological Diseases and Stroke NS077291 (WFE, JNS).

\section{Compliance with Ethical Standards}

Conflict of Interest The authors indicate no conflict of interest with the subject matter of this review. 
Human and Animal Rights and Informed Consent This article does not contain any studies with human or animal subjects performed by any of the authors.

\section{References}

1. Abbott NJ. Blood-brain barrier structure and function and the challenges for CNS drug delivery. J Inherit Metab Dis. 2013;36(3):437-49. doi:10.1007/s10545-013-9608-0.

2. Agarwal S, Hartz AM, Elmquist WF, Bauer B. Breast cancer resistance protein and P-glycoprotein in brain cancer: two gatekeepers team up. Curr Pharm Des. 2011a;17(26):2793-802.

3. Agarwal S, Sane R, Oberoi R, Ohlfest JR, Elmquist WF. Delivery of molecularly targeted therapy to malignant glioma, a disease of the whole brain. Expert Rev Mol Med. 2011b;13:e17. doi:10.1017 /S1462399411001888.

4. Agarwala SS, Kirkwood JM, Gore M, Dreno B, Thatcher N, Czarnetski B, et al. Temozolomide for the treatment of brain metastases associated with metastatic melanoma: a phase II study. J Clin Oncol. 2004;22(11):2101-7. doi:10.1200/JCO.2004.11.044.

5. Ajithkumar T, Parkinson C, Fife K, Corrie P, Jefferies S. Evolving treatment options for melanoma brain metastases. Lancet Oncol. 2015;16(13):e486-97. doi:10.1016/S1470-2045(15)00141-2.

6. Aplin AE, Kaplan FM, Shao Y. Mechanisms of resistance to RAF inhibitors in melanoma. J Invest Dermatol. 2011;131(9):1817-20. doi:10.1038/jid.2011.147.

7. Atefi M, Titz B, Avramis E, Ng C, Wong DJ, Lassen A, et al. Combination of pan-RAF and MEK inhibitors in NRAS mutant melanoma. Mol Cancer. 2015;14:27. doi:10.1186/s12943-0150293-5.

8. Atkins MB, Kunkel L, Sznol M, Rosenberg SA. High-dose recombinant interleukin-2 therapy in patients with metastatic melanoma: long-term survival update. Cancer J Sci Am. 2000;6(Suppl 1):S11-4.

9. Atkins MB, Lotze MT, Dutcher JP, Fisher RI, Weiss G, Margolin $\mathrm{K}$, et al. High-dose recombinant interleukin 2 therapy for patients with metastatic melanoma: analysis of 270 patients treated between 1985 and 1993. J Clin Oncol. 1999;17(7):2105-16.

10. Barkan D, Chambers AF. Beta1-integrin: a potential therapeutic target in the battle against cancer recurrence. Clin Cancer Res. 2011;17(23):7219-23. doi:10.1158/1078-0432.CCR-11-0642.

11. Bindal AK, Bindal RK, Hess KR, Shiu A, Hassenbusch SJ, Shi $\mathrm{WM}$, et al. Surgery versus radiosurgery in the treatment of brain metastasis. J Neurosurg. 1996;84(5):748-54. doi:10.3171 /jns. 1996.84.5.0748.

12. Cancer Facts and Figures 2016. American Cancer Society. 2016.

13. Cancer Genome Atlas N. Genomic classification of cutaneous melanoma. Cell. 2015;161(7):1681-96. doi:10.1016/j. cell.2015.05.044

14. Carlson BL, Pokorny JL, Schroeder MA, Sarkaria JN. Establishment, maintenance and in vitro and in vivo applications of primary human glioblastoma multiforme (GBM) xenograft models for translational biology studies and drug discovery. Curr Protoc Pharmacol. 2011;Chapter 14:Unit 14 6. doi:10.1002 /0471141755.ph1416s52.

15. Chapman PB, Hauschild A, Robert C, Haanen JB, Ascierto P, Larkin J, et al. Improved survival with vemurafenib in melanoma with BRAF V600E mutation. N Engl J Med. 2011;364(26):250716. doi:10.1056/NEJMoa1103782.

16. Chartrain M, Riond J, Stennevin A, Vandenberghe I, Gomes B, Lamant L, et al. Melanoma chemotherapy leads to the selection of ABCB5-expressing cells. PLoS One. 2012;7(5):e36762. doi:10.1371/journal.pone.0036762.
17. Choo EF, Ly J, Chan J, Shahidi-Latham SK, Messick K, Plise E, et al. Role of P-glycoprotein on the brain penetration and brain pharmacodynamic activity of the MEK inhibitor cobimetinib. Mol Pharm. 2014;11(11):4199-207. doi:10.1021/mp500435s.

18. Chu MB, Fesler MJ, Armbrecht ES, Fosko SW, Hsueh E, Richart JM. High-dose interleukin-2 (HD IL-2) therapy should be considered for treatment of patients with melanoma brain metastases. Chemother Res Pract. 2013;2013:726925. doi:10.1155/2013 1726925.

19. Cohen JV, Alomari AK, Vortmeyer AO, Jilaveanu LB, Goldberg SB, Mahajan A et al. Melanoma brain metastasis pseudoprogression after pembrolizumab treatment. Cancer Immunol Res 2016;4(3):179-182. doi:10.1158/2326-6066.CIR15-0160.

20. Rasco DW. MLN2480, an investigational oral pan-RAF kinase inhibitor, in patients (pts) with relapsed or refractory solid tumors: phase I study. 2013 ASCO Annual Meeting 2013.

21. Damsky WE, Rosenbaum LE, Bosenberg M. Decoding melanoma metastasis. Cancers (Basel). 2010;3(1):126-63. doi:10.3390 /cancers3010126.

22. Damsky WE, Theodosakis N, Bosenberg M. Melanoma metastasis: new concepts and evolving paradigms. Oncogene. 2014;33(19):2413-22. doi:10.1038/onc.2013.194.

23. Dana-Farber CI. Remission of Jimmy Carter's melanoma shows potential of immunotherapy for cancer. 2015.

24. Das R, Verma R, Sznol M, Boddupalli CS, Gettinger SN, Kluger $\mathrm{H}$, et al. Combination therapy with anti-CTLA-4 and anti-PD-1 leads to distinct immunologic changes in vivo. J Immunol. 2015;194(3):950-9. doi:10.4049/jimmunol.1401686.

25. Davies H, Bignell GR, Cox C, Stephens P, Edkins S, Clegg S, et al. Mutations of the BRAF gene in human cancer. Nature. 2002;417(6892):949-54. doi:10.1038/nature00766.

26. Davies MA, Liu P, McIntyre S, Kim KB, Papadopoulos N, Hwu WJ, et al. Prognostic factors for survival in melanoma patients with brain metastases. Cancer. 2011;117(8):1687-96. doi:10.1002/cncr.25634.

27. de Gooijer MC, Zhang P, Thota N, Mayayo-Peralta I, Buil LC, Beijnen JH, et al. P-glycoprotein and breast cancer resistance protein restrict the brain penetration of the CDK4/6 inhibitor palbociclib. Investig New Drugs. 2015;33(5):1012-9. doi:10.1007/s10637-015-0266-y.

28. Di Giacomo AM, Ascierto PA, Pilla L, Santinami M, Ferrucci PF, Giannarelli D, et al. Ipilimumab and fotemustine in patients with advanced melanoma (NIBIT-M1): an open-label, single-arm phase 2 trial. Lancet Oncol. 2012;13(9):879-86. doi:10.1016 /S1470-2045(12)70324-8.

29. Di Giacomo AM, Ascierto PA, Queirolo P, Pilla L, Ridolfi R, Santinami M, et al. Three-year follow-up of advanced melanoma patients who received ipilimumab plus fotemustine in the Italian Network for Tumor Biotherapy (NIBIT)-M1 phase II study. Ann Oncol. 2015;26(4):798-803. doi:10.1093/annonc/mdu577.

30. Di Giacomo AM, Margolin K. Immune checkpoint blockade in patients with melanoma metastatic to the brain. Semin Oncol. 2015;42(3):459-65. doi:10.1053/j.seminoncol.2015.02.006.

31. Dummer R, Goldinger SM, Turtschi CP, Eggmann NB, Michielin $\mathrm{O}$, Mitchell L, et al. Vemurafenib in patients with BRAF(V600) mutation-positive melanoma with symptomatic brain metastases: final results of an open-label pilot study. Eur J Cancer. 2014;50(3): 611-21. doi:10.1016/j.ejca.2013.11.002.

32. Durmus S, Hendrikx JJ, Schinkel AH. Apical ABC transporters and cancer chemotherapeutic drug disposition. Adv Cancer Res. 2015;125:1-41. doi:10.1016/bs.acr.2014.10.001.

33. Durmus S, Sparidans RW, Wagenaar E, Beijnen JH, Schinkel AH. Oral availability and brain penetration of the B-RAFV600E inhibitor vemurafenib can be enhanced by the P-GLYCOprotein $(\mathrm{ABCB} 1)$ and breast cancer resistance protein $(\mathrm{ABCG} 2)$ inhibitor 
elacridar. Mol Pharm. 2012;9(11):3236-45. doi:10.1021 /mp3003144.

34. Eigentler TK, Figl A, Krex D, Mohr P, Mauch C, Rass K, et al. Number of metastases, serum lactate dehydrogenase level, and type of treatment are prognostic factors in patients with brain metastases of malignant melanoma. Cancer. 2011;117(8):1697703. doi:10.1002/cncr.25631.

35. Eisele SC, Gill CM, Shankar GM, Brastianos PK. PLEKHA5: a key to unlock the blood-brain barrier? Clin Cancer Res. 2015;21(9):1978-80. doi:10.1158/1078-0432.CCR-14-2604.

36. Engelhardt B, Coisne C. Fluids and barriers of the CNS establish immune privilege by confining immune surveillance to a twowalled castle moat surrounding the CNS castle. Fluids Barriers CNS. 2011;8(1):4. doi:10.1186/2045-8118-8-4.

37. Falchook GS, Long GV, Kurzrock R, Kim KB, Arkenau TH, Brown MP, et al. Dabrafenib in patients with melanoma, untreated brain metastases, and other solid tumours: a phase 1 doseescalation trial. Lancet. 2012;379(9829):1893-901. doi:10.1016 /S0140-6736(12)60398-5.

38. Fidler IJ. The pathogenesis of cancer metastasis: the 'seed and soil' hypothesis revisited. Nat Rev Cancer. 2003;3(6):453-8. doi: $10.1038 /$ nrc1098.

39. Fidler IJ. The role of the organ microenvironment in brain metastasis. Semin Cancer Biol. 2011;21(2):107-12. doi:10.1016/j. semcancer.2010.12.009.

40. Fidler IJ. The biology of brain metastasis: challenges for therapy. Cancer J. 2015;21(4):284-93. doi:10.1097 /PPO.0000000000000126.

41. Fidler IJ, Balasubramanian K, Lin Q, Kim SW, Kim SJ. The brain microenvironment and cancer metastasis. Mol Cells. 2010;30(2): 93-8. doi:10.1007/s10059-010-0133-9.

42. Fife KM, Colman MH, Stevens GN, Firth IC, Moon D, Shannon $\mathrm{KF}$, et al. Determinants of outcome in melanoma patients with cerebral metastases. J Clin Oncol. 2004;22(7):1293-300. doi:10.1200/JCO.2004.08.140.

43. Flaherty KT, Infante JR, Daud A, Gonzalez R, Kefford RF, Sosman J, et al. Combined BRAF and MEK inhibition in melanoma with BRAF V600 mutations. N Engl J Med. 2012a. doi:10.1056/NEJMoa1210093.

44. Flaherty KT, Robert C, Hersey P, Nathan P, Garbe C, Milhem M, et al. Improved survival with MEK inhibition in BRAF-mutated melanoma. N Engl J Med. 2012b;367(2):107-14. doi:10.1056 /NEJMoa1203421.

45. Frenard C, Peuvrel L, Jean MS, Brocard A, Knol AC, Nguyen JM, et al. Development of brain metastases in patients with metastatic melanoma while receiving ipilimumab. J Neuro-Oncol. 2016;126(2):355-60. doi:10.1007/s11060-015-1977-9.

46. Gallego Perez-Larraya J, Hildebrand J. Brain metastases. Handb Clin Neurol. 2014;121:1143-57. doi:10.1016/B978-0-70204088-7.00077-8.

47. Gerstner ER, Fine RL. Increased permeability of the blood-brain barrier to chemotherapy in metastatic brain tumors: establishing a treatment paradigm. J Clin Oncol. 2007;25(16):2306-12. doi:10.1200/JCO.2006.10.0677.

48. Gibney GT, Forsyth PA, Sondak VK. Melanoma in the brain: biology and therapeutic options. Melanoma Res. 2012;22(3): 177-83. doi:10.1097/CMR.0b013e328352dbef.

49. Girotti MR, Lopes F, Preece N, Niculescu-Duvaz D, Zambon A, Davies L, et al. Paradox-breaking RAF inhibitors that also target SRC are effective in drug-resistant BRAF mutant melanoma. Cancer Cell. 2015;27(1):85-96. doi:10.1016/j.ccell.2014.11.006.

50. Goel VK, Lazar AJ, Warneke CL, Redston MS, Haluska FG. Examination of mutations in BRAF, NRAS, and PTEN in primary cutaneous melanoma. J Invest Dermatol. 2006;126(1):154-60. doi:10.1038/sj.jid.5700026.
51. Gorantla V, Kirkwood JM, Tawbi HA. Melanoma brain metastases: an unmet challenge in the era of active therapy. Curr Oncol Rep. 2013;15(5):483-91. doi:10.1007/s11912-013-0335-3.

52. Goyal S, Silk AW, Tian S, Mehnert J, Danish S, Ranjan S, et al. Clinical management of multiple melanoma brain metastases: a systematic review. JAMA Oncol. 2015;1(5):668-76. doi:10.1001 /jamaoncol.2015.1206.

53. Guirguis LM, Yang JC, White DE, Steinberg SM, Liewehr DJ, Rosenberg SA, et al. Safety and efficacy of high-dose interleukin2 therapy in patients with brain metastases. J Immunother. 2002;25(1):82-7.

54. Gupta G, Robertson AG, MacKie RM. Cerebral metastases of cutaneous melanoma. Br J Cancer. 1997;76(2):256-9.

55. Hauschild A, Grob JJ, Demidov LV, Jouary T, Gutzmer R, Millward M, et al. Dabrafenib in BRAF-mutated metastatic melanoma: a multicentre, open-label, phase 3 randomised controlled trial. Lancet. 2012;380(9839):358-65. doi:10.1016/S0140-6736 (12)60868-X.

56. Hiniker SM, Maecker HT, Knox SJ. Predictors of clinical response to immunotherapy with or without radiotherapy. J Radiat Oncol. 2015;4:339-45. doi:10.1007/s13566-015-0219-2.

57. Hocker T, Tsao H. Ultraviolet radiation and melanoma: a systematic review and analysis of reported sequence variants. Hum Mutat. 2007;28(6):578-88. doi:10.1002/humu.20481.

58. Hodi FS, O'Day SJ, McDermott DF, Weber RW, Sosman JA, Haanen JB, et al. Improved survival with ipilimumab in patients with metastatic melanoma. N Engl J Med. 2010;363(8):711-23. doi:10.1056/NEJMoa1003466.

59. Hodi FS, Oble DA, Drappatz J, Velazquez EF, Ramaiya N, Ramakrishna N, et al. CTLA-4 blockade with ipilimumab induces significant clinical benefit in a female with melanoma metastases to the CNS. Nat Clin Pract Oncol. 2008;5(9):557-61. doi:10.1038 /ncponc1183.

60. Hodis E, Watson IR, Kryukov GV, Arold ST, Imielinski M, Theurillat JP, et al. A landscape of driver mutations in melanoma. Cell. 2012;150(2):251-63. doi:10.1016/j.cell.2012.06.024.

61. Holdhoff M, Yovino SG, Boadu O, Grossman SA. Blood-based biomarkers for malignant gliomas. J Neuro-Oncol. 2013;113(3): 345-52. doi:10.1007/s11060-013-1144-0.

62. Huang RY, Neagu MR, Reardon DA, Wen PY. Pitfalls in the neuroimaging of glioblastoma in the era of antiangiogenic and immuno/targeted therapy - detecting illusive disease, defining response. Front Neurol. 2015;6:33. doi:10.3389/fneur.2015.00033.

63. Izraely S, Sagi-Assif O, Klein A, Meshel T, Ben-Menachem S, Zaritsky A, et al. The metastatic microenvironment: Claudin-1 suppresses the malignant phenotype of melanoma brain metastasis. Int J Cancer. 2015;136(6):1296-307. doi:10.1002/ijc.29090.

64. Jia W, Lu R, Martin TA, Jiang WG. The role of claudin-5 in bloodbrain barrier (BBB) and brain metastases (review). Mol Med Rep. 2014;9(3):779-85. doi:10.3892/mmr.2013.1875.

65. Joyce JA, Pollard JW. Microenvironmental regulation of metastasis. Nat Rev Cancer. 2009;9(4):239-52. doi:10.1038/nrc2618.

66. Kalaaji AN. Cytokine therapy in advanced melanoma. J Drugs Dermatol. 2007;6(4):374-8.

67. Karasarides M, Chiloeches A, Hayward R, Niculescu-Duvaz D, Scanlon I, Friedlos F, et al. B-RAF is a therapeutic target in melanoma. Oncogene. 2004;23(37):6292-8. doi:10.1038/sj. onc. 1207785.

68. Kaufman HL, Ruby CE, Hughes T, Slingluff Jr CL. Current status of granulocyte-macrophage colony-stimulating factor in the immunotherapy of melanoma. J Immunother Cancer. 2014;2:11. doi:10.1186/2051-1426-2-11.

69. Kerbel RS. A decade of experience in developing preclinical models of advanced- or early-stage spontaneous metastasis to study antiangiogenic drugs, metronomic chemotherapy, and the 
tumor microenvironment. Cancer J. 2015;21(4):274-83. doi:10.1097/PPO.0000000000000134.

70. Kolar GR, Miller-Thomas MM, Schmidt RE, Simpson JR, Rich KM, Linette GP. Neoadjuvant treatment of a solitary melanoma brain metastasis with vemurafenib. J Clin Oncol. 2013;31(3):e40 3. doi:10.1200/JCO.2012.43.7061.

71. Kruser TJ, Mehta MP, Robins HI. Pseudoprogression after glioma therapy: a comprehensive review. Expert Rev Neurother. 2013;13(4):389-403. doi:10.1586/ern.13.7.

72. Langley RR, Fidler IJ. Tumor cell-organ microenvironment interactions in the pathogenesis of cancer metastasis. Endocr Rev. 2007;28(3):297-321. doi:10.1210/er.2006-0027.

73. Larkin J, Ascierto PA, Dreno B, Atkinson V, Liszkay G, Maio M, et al. Combined vemurafenib and cobimetinib in BRAF-mutated melanoma. N Engl J Med. 2014;371(20):1867-76. doi:10.1056 /NEJMoa1408868.

74. Lee MS, Helms TL, Feng N, Gay J, Chang QE, Tian F, et al. Efficacy of the combination of MEK and CDK4/6 inhibitors in vitro and in vivo in KRAS mutant colorectal cancer models. Oncotarget. 2016. doi:10.18632/oncotarget.9153.

75. Lonser RR, Song DK, Klapper J, Hagan M, Auh S, Kerr PB, et al. Surgical management of melanoma brain metastases in patients treated with immunotherapy. J Neurosurg. 2011;115(1):30-6. doi:10.3171/2011.3.JNS091107.

76. Luo Y, Ellis LZ, Dallaglio K, Takeda M, Robinson WA, Robinson $\mathrm{SE}$, et al. Side population cells from human melanoma tumors reveal diverse mechanisms for chemoresistance. J Invest Dermatol. 2012;132(10):2440-50. doi:10.1038/jid.2012.161.

77. Margolin K, Ernstoff MS, Hamid O, Lawrence D, McDermott D, Puzanov I, et al. Ipilimumab in patients with melanoma and brain metastases: an open-label, phase 2 trial. Lancet Oncol. 2012;13(5): 459-65. doi:10.1016/S1470-2045(12)70090-6.

78. McCubrey JA, Milella M, Tafuri A, Martelli AM, Lunghi P, Bonati A, et al. Targeting the Raf/MEK/ERK pathway with small-molecule inhibitors. Curr Opin Investig Drugs. 2008;9(6): 614-30.

79. McDermott D, Lebbe C, Hodi FS, Maio M, Weber JS, Wolchok JD, et al. Durable benefit and the potential for long-term survival with immunotherapy in advanced melanoma. Cancer Treat Rev. 2014;40(9):1056-64. doi:10.1016/j.ctrv.2014.06.012.

80. McWilliams RR, Brown PD, Buckner JC, Link MJ, Markovic SN. Treatment of brain metastases from melanoma. Mayo Clin Proc. 2003;78(12):1529-36. doi:10.4065/78.12.1529.

81. Melero I, Grimaldi AM, Perez-Gracia JL, Ascierto PA. Clinical development of immunostimulatory monoclonal antibodies and opportunities for combination. Clin Cancer Res. 2013;19(5): 997-1008. doi:10.1158/1078-0432.CCR-12-2214.

82. Miller DM, Flaherty KT. Cyclin-dependent kinases as therapeutic targets in melanoma. Pigment Cell Melanoma Res. 2014;27(3): 351-65. doi:10.1111/pcmr.12211.

83. Mittapalli RK, Vaidhyanathan S, Dudek AZ, Elmquist WF. Mechanisms limiting distribution of the threonine-protein kinase B-RaF(V600E) inhibitor dabrafenib to the brain: implications for the treatment of melanoma brain metastases. J Pharmacol Exp Ther. 2013;344(3):655-64. doi:10.1124 /jpet.112.201475.

84. Mittapalli RK, Vaidhyanathan S, Sane R, Elmquist WF. Impact of P-glycoprotein $(\mathrm{ABCB} 1)$ and breast cancer resistance protein $(\mathrm{ABCG})$ on the brain distribution of a novel BRAF inhibitor: vemurafenib (PLX4032). J Pharmacol Exp Ther. 2012;342(1): 33-40. doi:10.1124/jpet.112.192195.

85. Nakamura A, Arita T, Tsuchiya S, Donelan J, Chouitar J, Carideo E, et al. Antitumor activity of the selective pan-RAF inhibitor TAK-632 in BRAF inhibitor-resistant melanoma. Cancer Res. 2013;73(23):7043-55. doi:10.1158/0008-5472.CAN-13-1825.
86. Nikolaou VA, Stratigos AJ, Flaherty KT, Tsao H. Melanoma: new insights and new therapies. J Invest Dermatol. 2012;132(3 Pt 2): 854-63. doi:10.1038/jid.2011.421.

87. Ohtsuki S, Terasaki T. Contribution of carrier-mediated transport systems to the blood-brain barrier as a supporting and protecting interface for the brain; importance for CNS drug discovery and development. Pharm Res. 2007;24(9):1745-58. doi:10.1007 /s11095-007-9374-5.

88. Ott PA, Hodi FS, Robert C. CTLA-4 and PD-1/PD-L1 blockade: new immunotherapeutic modalities with durable clinical benefit in melanoma patients. Clin Cancer Res. 2013;19(19):5300-9. doi:10.1158/1078-0432.CCR-13-0143.

89. Parrish KE, Pokorny J, Mittapalli RK, Bakken K, Sarkaria JN, Elmquist WF. Efflux transporters at the blood-brain barrier limit delivery and efficacy of cyclin-dependent kinase 4/6 inhibitor palbociclib (PD-0332991) in an orthotopic brain tumor model. J Pharmacol Exp Ther. 2015a;355(2):264-71. doi:10.1124 /jpet.115.228213.

90. Parrish KE, Sarkaria JN, Elmquist WF. Improving drug delivery to primary and metastatic brain tumors: strategies to overcome the blood-brain barrier. Clin Pharmacol Ther. 2015b;97(4):336-46. doi:10.1002/cpt.71.

91. Patel BG, Ahmed KA, Johnstone PA, Yu HM, Etame AB. Initial experience with combined BRAF and MEK inhibition with stereotactic radiosurgery for BRAF mutant melanoma brain metastases. Melanoma Res. 2016. doi:10.1097 /CMR.0000000000000250.

92. Peng SB, Henry JR, Kaufman MD, Lu WP, Smith BD, Vogeti S, et al. Inhibition of RAF isoforms and active dimers by LY3009120 leads to anti-tumor activities in RAS or BRAF mutant cancers. Cancer Cell. 2015;28(3):384-98. doi:10.1016/j. ccell.2015.08.002.

93. Persidsky Y, Ramirez SH, Haorah J, Kanmogne GD. Blood-brain barrier: structural components and function under physiologic and pathologic conditions. J NeuroImmune Pharmacol. 2006;1(3): 223-36. doi:10.1007/s11481-006-9025-3.

94. Peuvrel L, Saint-Jean M, Quereux G, Brocard A, Khammari A, $\mathrm{Knol} \mathrm{AC}$, et al. Incidence and characteristics of melanoma brain metastases developing during treatment with vemurafenib. J Neuro-Oncol. 2014;120(1):147-54. doi:10.1007/s11060-0141533-z.

95. Pope WB, Djoukhadar I, Jackson A. Neuroimaging. Handb Clin Neurol. 2016;134:27-50. doi:10.1016/B978-0-12-8029978.00003-7.

96. Poulikakos PI, Zhang C, Bollag G, Shokat KM, Rosen N. RAF inhibitors transactivate RAF dimers and ERK signalling in cells with wild-type BRAF. Nature. 2010;464(7287):427-30. doi:10.1038/nature08902.

97. Powell S, Dudek AZ. Single-institution outcome of high-dose interleukin-2 (HD IL-2) therapy for metastatic melanoma and analysis of favorable response in brain metastases. Anticancer Res. 2009;29(10):4189-93.

98. Puhalla S, Elmquist W, Freyer D, Kleinberg L, Adkins C, Lockman P, et al. Unsanctifying the sanctuary: challenges and opportunities with brain metastases. Neuro-Oncology. 2015;17(5):639-51. doi:10.1093/neuonc/nov023.

99. Puzanov I, Burnett P, Flaherty KT. Biological challenges of BRAF inhibitor therapy. Mol Oncol. 2011;5(2):116-23. doi:10.1016/j. molonc.2011.01.005.

100. Quail DF, Joyce JA. Microenvironmental regulation of tumor progression and metastasis. Nat Med. 2013;19(11):1423-37. doi: $10.1038 / \mathrm{nm} .3394$.

101. Queirolo P, Spagnolo F, Ascierto PA, Simeone E, Marchetti P, Scoppola A, et al. Efficacy and safety of ipilimumab in patients with advanced melanoma and brain metastases. J Neuro-Oncol. 2014;118(1):109-16. doi:10.1007/s11060-014-1400-y. 
102. Radbruch A, Fladt J, Kickingereder P, Wiestler B, Nowosielski M, Baumer P, et al. Pseudoprogression in patients with glioblastoma: clinical relevance despite low incidence. Neuro-Oncology. 2015;17(1):151-9. doi:10.1093/neuonc/nou129.

103. Raizer JJ, Hwu WJ, Panageas KS, Wilton A, Baldwin DE, Bailey $\mathrm{E}$, et al. Brain and leptomeningeal metastases from cutaneous melanoma: survival outcomes based on clinical features. NeuroOncology. 2008;10(2):199-207. doi:10.1215/15228517-2007058.

104. Ribas A, Gonzalez R, Pavlick A, Hamid O, Gajewski TF, Daud A, et al. Combination of vemurafenib and cobimetinib in patients with advanced BRAF(V600)-mutated melanoma: a phase 1b study. Lancet Oncol. 2014;15(9):954-65. doi:10.1016/S14702045(14)70301-8.

105. Ribas A, Kefford R, Marshall MA, Punt CJ, Haanen JB, Marmol $\mathrm{M}$, et al. Phase III randomized clinical trial comparing tremelimumab with standard-of-care chemotherapy in patients with advanced melanoma. J Clin Oncol. 2013;31(5):616-22. doi:10.1200/JCO.2012.44.6112.

106. Ribas A, Kim K, Schuchter L, Gonzalez R, Pavlick AC, Weber JS, et al. BRIM-2: an open-label, multicenter phase II study of vemurafenib in previously treated patients with BRAF V600E mutation-positive metastatic melanoma. ASCO Annual Meeting; J Clin Oncol. 2011;29(suppl; abstr 8509). 2011.

107. Robert C, Thomas L, Bondarenko I, O'Day S, Weber J, Garbe C, et al. Ipilimumab plus dacarbazine for previously untreated metastatic melanoma. N Engl J Med. 2011;364(26):2517-26. doi:10.1056/NEJMoa1104621.

108. Rochet NM, Dronca RS, Kottschade LA, Chavan RN, Gorman B, Gilbertson JR, et al. Melanoma brain metastases and vemurafenib: need for further investigation. Mayo Clin Proc. 2012;87(10):97681. doi:10.1016/j.mayocp.2012.07.006.

109. Ruggeri BA, Camp F, Miknyoczki S. Animal models of disease: pre-clinical animal models of cancer and their applications and utility in drug discovery. Biochem Pharmacol. 2014;87(1):150 61. doi:10.1016/j.bcp.2013.06.020.

110. Salvati M, Frati A, D'Elia A, Pescatori L, Piccirilli M, Pietrantonio A, et al. Single brain metastases from melanoma: remarks on a series of 84 patients. Neurosurg Rev. 2012;35(2):211-7 discussion 7-8. doi:10.1007/s10143-011-0348-z.

111. Samatar AA, Poulikakos PI. Targeting RAS-ERK signalling in cancer: promises and challenges. Nat Rev Drug Discov. 2014;13(12):928-42. doi:10.1038/nrd4281.

112. Sanghera P, Perry J, Sahgal A, Symons S, Aviv R, Morrison M, et al. Pseudoprogression following chemoradiotherapy for glioblastoma multiforme. Can J Neurol Sci. 2010;37(1):36-42.

113. Schoenfeld JD, Mahadevan A, Floyd SR, Dyer MA, Catalano PJ, Alexander BM, et al. Ipilmumab and cranial radiation in metastatic melanoma patients: a case series and review. J Immunother Cancer. 2015;3:50. doi:10.1186/s40425-015-0095-8.

114. Serrone L, Zeuli M, Sega FM, Cognetti F. Dacarbazine-based chemotherapy for metastatic melanoma: thirty-year experience overview. J Exp Clin Cancer Res. 2000;19(1):21-34.

115. Seyedin SN, Schoenhals JE, Lee DA, Cortez MA, Wang X, Niknam S, et al. Strategies for combining immunotherapy with radiation for anticancer therapy. Immunotherapy. 2015;7(9):96780. doi:10.2217/imt.15.65.

116. Shaik N, Giri N, Pan G, Elmquist WF. P-glycoprotein-mediated active efflux of the anti-HIV1 nucleoside abacavir limits cellular accumulation and brain distribution. Drug Metab Dispos. 2007;35(11):2076-85. doi:10.1124/dmd.107.017723.

117. Shapiro DG, Samlowski WE. Management of melanoma brain metastases in the era of targeted therapy. J Skin Cancer. 2011;2011:845863. doi:10.1155/2011/845863.

118. Sharma P, Allison JP. The future of immune checkpoint therapy. Science. 2015a;348(6230):56-61. doi:10.1126/science.aaa8172.
119. Sharma P, Allison JP. Immune checkpoint targeting in cancer therapy: toward combination strategies with curative potential. Cell. 2015b;161(2):205-14. doi:10.1016/j.cell.2015.03.030.

120. Siegel RL, Miller KD, Jemal A. Cancer statistics, 2016. CA Cancer J Clin. 2016;66(1):7-30. doi:10.3322/caac.21332.

121. Sloan AE, Nock CJ, Einstein DB. Diagnosis and treatment of melanoma brain metastasis, a literature review. Cancer Control. 2009;16(3):248-55.

122. Smalley KS, Haass NK, Brafford PA, Lioni M, Flaherty KT, Herlyn M. Multiple signaling pathways must be targeted to overcome drug resistance in cell lines derived from melanoma metastases. Mol Cancer Ther. 2006;5(5):1136-44. doi:10.1158/15357163.MCT-06-0084.

123. Sosman J. A phase $1 \mathrm{~b} / 2$ study of LEE011 in combination with binimetinib (MEK162) in patients with NRAS mutant melanoma: early encouraging clinical activity. J Clin Oncol. 2014;32:5 s (suppl; abstr 9009). 2014.

124. Stewart DJ. A critique of the role of the blood-brain barrier in the chemotherapy of human brain tumors. J Neuro-Oncol. 1994;20(2):121-39.

125. Tas F. Metastatic behavior in melanoma: timing, pattern, survival, and influencing factors. J Oncol. 2012;2012:647684. doi:10.1155 /2012/647684.

126. Taylor EM. The impact of efflux transporters in the brain on the development of drugs for CNS disorders. Clin Pharmacokinet. 2002;41(2):81-92. doi:10.2165/00003088-200241020-00001.

127. Topalian SL, Hodi FS, Brahmer JR, Gettinger SN, Smith DC, McDermott DF, et al. Safety, activity, and immune correlates of anti-PD-1 antibody in cancer. N Engl J Med. 2012;366(26):244354. doi:10.1056/NEJMoa1200690.

128. Topalian SL, Sznol M, McDermott DF, Kluger HM, Carvajal RD, Sharfman WH, et al. Survival, durable tumor remission, and longterm safety in patients with advanced melanoma receiving nivolumab. J Clin Oncol. 2014;32(10):1020-30. doi:10.1200 /JCO.2013.53.0105.

129. Trunzer K, Pavlick AC, Schuchter L, Gonzalez R, McArthur GA, Hutson TE, et al. Pharmacodynamic effects and mechanisms of resistance to vemurafenib in patients with metastatic melanoma. J Clin Oncol. 2013;31(14):1767-74. doi:10.1200 /JCO.2012.44.7888.

130. Tsai J, Lee JT, Wang W, Zhang J, Cho H, Mamo S, et al. Discovery of a selective inhibitor of oncogenic B-Raf kinase with potent antimelanoma activity. Proc Natl Acad Sci U S A. 2008;105(8): 3041-6. doi:10.1073/pnas.0711741105.

131. Uchida Y, Ohtsuki S, Katsukura Y, Ikeda C, Suzuki T, Kamiie J, et al. Quantitative targeted absolute proteomics of human bloodbrain barrier transporters and receptors. J Neurochem. 2011;117(2):333-45. doi:10.1111/j.1471-4159.2011.07208.x.

132. Vaidhyanathan S, Mittapalli RK, Sarkaria JN, Elmquist WF. Factors influencing the CNS distribution of a novel MEK-1/2 inhibitor: implications for combination therapy for melanoma brain metastases. Drug Metab Dispos. 2014;42(8):1292-300. doi:10.1124/dmd.114.058339.

133. Vaidhyanathan S, Wilken-Resman B, Ma DJ, Parrish KE, Mittapalli RK, Carlson BL, et al. Factors influencing the central nervous system distribution of a novel phosphoinositide 3-kinase/ mammalian target of rapamycin inhibitor GSK2126458: implications for overcoming resistance with combination therapy for melanoma brain metastases. J Pharmacol Exp Ther. 2016;356(2): 251-9. doi:10.1124/jpet.115.229393.

134. Villanueva J, Vultur A, Lee JT, Somasundaram R, FukunagaKalabis M, Cipolla AK, et al. Acquired resistance to BRAF inhibitors mediated by a RAF kinase switch in melanoma can be overcome by cotargeting MEK and IGF-1R/PI3K. Cancer Cell. 2010;18(6):683-95. doi:10.1016/j.ccr.2010.11.023. 
135. Wagle N, Emery C, Berger MF, Davis MJ, Sawyer A, Pochanard $\mathrm{P}$, et al. Dissecting therapeutic resistance to RAF inhibition in melanoma by tumor genomic profiling. J Clin Oncol. 2011;29(22):3085-96. doi:10.1200/JCO.2010.33.2312.

136. Wagle N, Van Allen EM, Treacy DJ, Frederick DT, Cooper ZA, Taylor-Weiner A, et al. MAP kinase pathway alterations in BRAFmutant melanoma patients with acquired resistance to combined RAF/MEK inhibition. Cancer Discov. 2014;4(1):61-8. doi:10.1158/2159-8290.CD-13-0631.

137. Wan PT, Garnett MJ, Roe SM, Lee S, Niculescu-Duvaz D, Good $\mathrm{VM}$, et al. Mechanism of activation of the RAF-ERK signaling pathway by oncogenic mutations of B-RAF. Cell. 2004;116(6):855-67.

138. Whittaker SR, Cowley GS, Wagner S, Luo F, Root DE, Garraway LA. Combined pan-RAF and MEK inhibition overcomes multiple resistance mechanisms to selective RAF inhibitors. Mol Cancer Ther. 2015;14(12):2700-11. doi:10.1158/1535-7163.MCT-150136-T.

139. Wilhelm I, Molnar J, Fazakas C, Hasko J, Krizbai IA. Role of the blood-brain barrier in the formation of brain metastases. Int J Mol Sci. 2013;14(1):1383-411. doi:10.3390/ijms14011383.
140. Wolchok JD, Kluger H, Callahan MK, Postow MA, Rizvi NA, Lesokhin AM, et al. Nivolumab plus ipilimumab in advanced melanoma. N Engl J Med. 2013;369(2):122-33. doi:10.1056 /NEJMoa1302369.

141. Yadav V, Burke TF, Huber L, Van Horn RD, Zhang Y, Buchanan SG, et al. The CDK4/6 inhibitor LY2835219 overcomes vemurafenib resistance resulting from MAPK reactivation and cyclin D1 upregulation. Mol Cancer Ther. 2014;13(10):2253-63. doi:10.1158/1535-7163.MCT-140257.

142. Yadav V, Chen SH, Yue YG, Buchanan S, Beckmann RP, Peng SB. Co-targeting BRAF and cyclin dependent kinases 4/6 for BRAF mutant cancers. Pharmacol Ther. 2015;149:139-49. doi:10.1016/j.pharmthera.2014.12.003.

143. Zakrzewski J, Geraghty LN, Rose AE, Christos PJ, Mazumdar M, Polsky D, et al. Clinical variables and primary tumor characteristics predictive of the development of melanoma brain metastases and post-brain metastases survival. Cancer. 2011;117(8):1711-20. doi:10.1002 /encr.25643. 